Pacific Journal of Mathematics

ISOMORPHIC BANACH-STONE THEOREMS AND
ISOMORPHISMS WHICH ARE CLOSE TO ISOMETRIES 


\title{
ISOMORPHIC BANACH-STONE THEOREMS AND ISOMORPHISMS WHICH ARE CLOSE TO ISOMETRIES
}

\author{
EHRHARD BEHRENDS
}

\begin{abstract}
A Banach space $X$ is said to have the isomorphic Banach-Stone property if for locally compact Hausdorff spaces $K$ and $L$ one always can conclude that $K$ and $L$ are homeomorphic provided that the Banach spaces $C_{0}(K, X)$ and $C_{0}(L, X)$ (=the continuous $X$-valued functions on $K$ resp. $L$ which vanish at infinity) are isomorphic with sufficiently small Banach-Mazur distance.

Our main results are a characterization of the finite-dimensional spaces with this property, and we also get an abundance of new finiteand infinite-dimensional examples.

These results appear as corollaries to general theorems about isomorphisms between certain spaces of continuous vector-valued functions. They enable us also to conclude that, for certain spaces $X$, and all compact $K$ all isomorphisms $T$ on $C_{0}(K, X)$ with $(1 /(1+\tau))\|f\| \leq$ $\|T f\| \leq(1+\tau)\|f\|$ for small $\tau$ can be approximated by isometries.
\end{abstract}

1. Introduction. The classical Banach-Stone theorem asserts that two locally compact Hausdorff spaces $K$ and $L$ are homeomorphic if and only if the Banach spaces $C_{0} K$ and $C_{0} L$ are isometrically isomorphic $\left(C_{0} K=\right.$ the space of continuous scalar-valued functions which vanish at infinity, provided with the supremum norm). Several generalizations of this theorem have been studied in the literature. For example, there have been investigated Banach spaces $X$ such that "scalar-valued" can be replaced by " $X$-valued" in the assertion of the Banach-Stone theorem. $X$ is then said to have the Banach-Stone property. This generalization is well understood; the interested reader is referred to [4] and [5] where it is shown that in a sense which can be made precise one knows all possibilities to determine spaces with the Banach-Stone property.

Another generalization is due to Amir [2] and Cambern [9] who proved independently that in the assertion of the theorem one may replace " $C_{0} K$ and $C_{0} L$ are isometrically isomorphic" by "the Banach spaces $C_{0} K$ and $C_{0} L$ are isomorphic, and the Banach-Mazur distance is smaller than two". Later it was shown that also in this version 
one may replace the scalars by certain Banach spaces if the BanachMazur distance of the function spaces under consideration is sufficiently small. The most far-reaching results into this direction are those of Cambern [11] and Jarosz [15].

1.1. Definition. Let $X$ be a Banach space. We will say that $X$ has the isomorphic Banach-Stone property if there is a $\delta>0$ such that the following holds: whenever $K$ and $L$ are locally compact Hausdorff spaces such that there exists an isomorphism $T: C_{0}(K, X) \rightarrow C_{0}(L, X)$ (these are the continuous $X$-valued functions on $K$ resp. $L$ which vanish at infinity, and this function space is provided with the supremum norm), then $K$ and $L$ are homeomorphic provided that $\|T\|\left\|T^{-1}\right\| \leq$ $1+\delta$.

Cambern proved that uniformly convex spaces have this property, and by Jarosz's result a certain rather technical condition concerning the dual of $X$ is sufficient.

Given $K$ and $L$ and a homeomorphism $\varphi: K \rightarrow L$ one can try to define $T: C_{0}(K, X) \rightarrow C_{0}(L, X)$ by $(T f)(\varphi(k))=T_{k}[f(k)]$, where $T_{k}$ is an isomorphism from $X$ to $X$ for every $k$. A moment's reflection shows that $T$ will be an isomorphism with $\|T\|\left\|T^{-1}\right\|$ close to one if the $T_{k}$ have uniformly this property, and it even suffices that the $(T f)(\varphi(k))$ are close to the $T_{k}[f(k)]$. One might ask whether every isomorphism $T$ can be approximated in this way. To be more precise:

1.2. Definition. $X$ is said to have the strong isomorphic BanachStone property if for every $\varepsilon>0$ there is a $\delta>0$ such that the following holds: whenever $K, L$, and $T$ are given as in 1.1, then there exist a homeomorphism $\varphi: K \rightarrow L$ and a family $\left(T_{k}\right)_{k \in K}$ of isomorphisms on $X$ such that

$$
\begin{aligned}
& \left\|T_{k}\right\|\left\|T_{k}^{-1}\right\| \leq 1+\varepsilon \text { and } \\
& \left\|(T f)(\varphi(k))-T_{k}[f(k)]\right\| \leq \varepsilon
\end{aligned}
$$

for all $k \in K$ and all $f \in C_{0}(K, X)$ with $\|f\| \leq 1$.

There exist two fairly general approaches to decide whether a given $X$ satisfies 1.1 or 1.2. They are completely independent, but both rely on an isomorphic adaption of certain concepts of $M$-structure theory (which gave the most far-reaching results in the isometrical case). The first approach depends on the behaviour of certain $M$-ideals under isomorphisms. Using this it can be shown that every $X$ such that $l_{2}^{1}$ (the two-dimensional $L^{1}$-space) is bounded away uniformly from every two-dimensional subspace of $X^{\prime}$ (=the dual of $X$ ) has the 
isomorphic Banach-Stone property, a result which will be published in a joint paper of $M$. Cambern and the author [7].

Here we will study another approach which depends only in part on $M$-structure (namely on the behaviour of certain operators which are just the isomorphic variants of multipliers). In cases where both [7] and the present results apply the admissible $\delta$ 's which are determined by [7] are usually larger. In the present paper, however, we are provided also with spaces satisfying 1.2 , and in the finite-dimensional case we even obtain necessary and sufficient conditions.

In contrast to the isometric vector-valued theory several interesting questions are open for isomorphic vector-valued Banach-Stone theorems. In particular there is nothing known about the range of the possible constants $\delta$ for a Banach space $X$ with the isomorphic BanachStone property. The only exception is the case of one-dimensional $X$ : here precisely the $\delta<1$ are admissible ([10], [12]).

Our results will be derived as special cases of a much more general theory concerning isomorphisms between certain spaces of continuous vector-valued functions. To be more precise, we start with

1.4. Definition. Let $K_{1}, \ldots, K_{r}, L_{1}, \ldots, L_{s}$ be locally compact Hausdorff spaces and $X_{1}, \ldots, X_{r}, Y_{1}, \ldots, Y_{s}$ Banach spaces.

(i) $C_{0}\left(K_{1}, \ldots, K_{r} ; X_{1}, \ldots, X_{r}\right)$ denotes the space $\prod_{\rho=1}^{r \infty} C_{0}\left(K_{\rho}, X_{\rho}\right)$, the $L^{\infty}$-direct product of the $C_{0}\left(K_{\rho}, X_{\rho}\right)$ (this is just the product of these spaces, provided with the supremum norm). The elements will be regarded as "functions" $f$ defined on $K:=K_{1} \cup \cdots \cup K_{r}$, where for $k \in K_{\rho}$ the element $f(k)$ lies in $X_{\rho}$.

(ii) Let $\tau \geq 0$ and

$$
T: C_{0}\left(K_{1}, \ldots, K_{r} ; X_{1}, \ldots, X_{r}\right) \rightarrow C_{0}\left(L_{1}, \ldots, L_{s} ; Y_{1}, \ldots, Y_{s}\right)
$$

be an isomorphism. $T$ will be called a $\tau$-isomorphism if

$$
\frac{1}{1+\tau}\|f\| \leq\|T f\| \leq(1+\tau)\|f\|
$$

for every $f$ (clearly every isomorphism $T$ is - up to a constant-a $\tau$ isomorphism for a suitable $\tau$, and we may assume that $\|T\|\left\|T^{-1}\right\|=$ $(1+\tau)^{2}$; the advantage of considering $\tau$-isomorphisms lies in the fact $T$ and $T^{-1}$ satisfy the same norm conditions so that in the sequel one only will have to prove the assertions concerning $T$; those concerning $T^{-1}$ will then be true by symmetry).

We now are going to explain the results in some detail. In $\S 2$ we assign to each finite family $X_{1}, \ldots, X_{r}$ of Banach spaces a number 
$\alpha\left(X_{1}, \ldots, X_{r}\right)$. The definition is rather technical, and there is certainly no hope to calculate precise values in the general situation. However, we are able to prove the following:

TheOREM (TH. 2.4). Suppose that $\alpha\left(X_{1}, \ldots, X_{r}\right)+\alpha\left(Y_{1}, \ldots, Y_{s}\right)<1$. Then there exists a $\tau>0$ such that the existence of a $\tau$-isomorphism

$$
T: C_{0}\left(K_{1}, \ldots, K_{r} ; X_{1}, \ldots, X_{r}\right) \rightarrow C_{0}\left(L_{1}, \ldots, L_{s} ; Y_{1}, \ldots, Y_{s}\right)
$$

implies that there is a homeomorphism

$$
\varphi: K_{1} \dot{\cup} \cdots \dot{\cup} K_{r} \rightarrow L_{1} \dot{\cup} \cdots \dot{U} L_{s} .
$$

We even can show more. If we denote, for Banach spaces $X$ and $Y$, the Banach-Mazur distance

$$
\inf \left\{\|T\|\left\|T^{-1}\right\| T: X \rightarrow Y \text { an isomorphism }\right\}
$$

by $d(X, Y)$, then we have the

TheOREM (TH. 2.5). Suppose that $d\left(X_{\rho}, X_{\rho^{\prime}}\right), d\left(Y_{\sigma}, Y_{\sigma^{\prime}}\right)>1$ for all $\rho \neq \rho^{\prime}, \sigma \neq \sigma^{\prime}$. We further assume that $\alpha\left(X_{1}, \ldots, X_{r}\right)$ and $\alpha\left(Y_{1}, \ldots, Y_{s}\right)$ are sufficiently small. Then there exists $a \tau>0$ such that the existence of a $\tau$-isomorphism implies that $r=s$, that-up to rearrangement- $K_{\rho}$ and $L_{\rho}$ are homeomorphic, and $d\left(X_{\rho}, Y_{\rho}\right)$ is close to one for every $\rho$.

Of course these theorems are of no interest as long as we have no possibilities to give estimates for the constants $\alpha\left(X_{1}, \ldots, X_{r}\right)$, $\alpha\left(Y_{1}, \ldots, Y_{s}\right)$. We develop two possibilities to get such estimates. For the first one we introduce a new constant which measures, in a sense, how large the segments in the unit sphere are:

1.5. Definition. Let $X$ be a Banach space. By $\alpha_{1}(X)$ we denote the infimum of those numbers $\alpha>0$ for which there exists an $\varepsilon>0$ such that the following holds: if $x_{0}, x$ are vectors in $X$ with

$$
\begin{gathered}
1-\varepsilon \leq\left\|x_{0}\right\| \leq 1+\varepsilon \\
\left\|x_{0}+\Theta x\right\| \leq 1+\varepsilon \text { for all scalars } \Theta \text { with }|\Theta|=1,
\end{gathered}
$$

then $\|x\| \leq \alpha$.

In the case of finite-dimensional spaces $\alpha_{1}(X)$ is just the maximal radius of one-dimensional balls contained in the unit sphere.

$\alpha_{1}(X)=0$ just means that $X$ is uniformly convex (which has to be understood in the sense of [14] for complex spaces). 
The second possibility uses isomorphic variants of multipliers:

1.6. Definition. Let $X$ be a Banach space and $R: X \rightarrow X$ an operator

(i) Suppose first that $\|R\| \leq 1$. Then $R$ is called a multiplier (or $M$-bounded) if

$$
\left\|R x-x_{0}\right\| \leq 1
$$

whenever $x_{0}, x$ are vectors such that $\left\|\Theta x-x_{0}\right\| \leq 1$ for all scalars $\Theta$ with $|\Theta|=1$. Operators with $\|R\|>1$ will be called multipliers if $R /\|R\|$ has the above property.

The collection of all multipliers is denoted by $\operatorname{Mult}(X)$ (the $m u l$ tiplier of $X$ ), and this set is said to be trivial if it contains only the operators $\mathbf{K} \cdot \mathbf{I d}$; as usual $\mathbf{K}$ stands for the scalar field, $K=\mathbf{R}$ or $\mathbf{K}=\mathbf{C}$.

(ii) For $\varepsilon \geq 0$ we say that $R$ belongs to $\operatorname{Mult}_{\varepsilon}(X)$ if $R$ satisfies the following condition:

$$
\left\|R x-x_{0}\right\| \leq 1+\varepsilon
$$

for all vectors with $\left\|\Theta x-x_{0}\right\| \leq 1$ (all $\Theta$ with $\left.|\Theta|=1\right)$.

Note. For a survey of properties of $\operatorname{Mult}(X)$ the reader is referred to [1], [4], and [6]. For our purposes it is important to note that there are many classes of spaces known where $\operatorname{Mult}(X)$ is trivial, e.g.

$X$ strictly convex [16], [19]

$X$ smooth [6]

$X=L^{1}(\mu)$, and $\operatorname{dim} X>2[6]$.

$X$ is finite-dimensional and contains no nontrivial $M$-summand (i.e. no subspace $J$ different from $\{0\}$ and $X$ with an $L^{\infty}$-complement; [6]).

Trivially $\operatorname{Mult}_{0}(X)$ consists of the $R$ in $\operatorname{Mult}(X)$ with $\|R\| \leq 1$. We need to know how close the $R \in \operatorname{Mult}_{\varepsilon}(X)$, which behave nearly as multipliers, are to the trivial elements of $\operatorname{Mult}(X)$, the operators K Id. This gives rise to the next definition; there we will use the symbol $(M)_{\alpha}$ to denote, for a subset $M$ of a normed space, the set $\{x \mid$ there exists an $m \in M$ with $\|x-m\| \leq \alpha\}$.

1.7. Definition. $\alpha_{2}(X)$ denotes the infimum of those numbers $\alpha$ for which there is an $\varepsilon>0$ such that

$$
\operatorname{Mult}_{\varepsilon}(X) \subset(\mathbf{K} \text { Id })_{\alpha} .
$$

A systematic study of $\operatorname{Mult}_{\varepsilon}(X)$ and $\alpha_{2}(X)$ is not intended in this paper. We only collect together in $\S 3$ those properties which are of 
importance for our purposes. In particular we prove (Th. 3.2 and Th. 3.3):

\section{THEOREM.}

(i) If $X$ is finite-dimensional and $\operatorname{Mult}(X)$ is trivial then $\alpha_{2}(X)=0$.

(ii) $\alpha_{2}(X)=0$ for every uniformly smooth space.

Our main result, by which we are in a position to apply the investigations of $\S 2$, is then the following theorem. Its proof and the first consequences are discussed in $\S 4$ :

Theorem. Let $X_{1}, \ldots, X_{r}$ be Banach spaces. Then

$$
\alpha\left(X_{1}, \ldots, X_{r}\right) \leq \max _{\rho} \min \left\{\alpha_{1}\left(X_{\rho}\right), 2 \alpha_{2}\left(X_{\rho}\right)\right\} .
$$

It is then fairly easy to derive various isomorphic Banach-Stone theorems. In $\S 5$ we prove:

- uniformly convex and uniformly smooth spaces have the strong isomorphic Banach-Stone property;

- let $X$ be finite-dimensional; we write $X$ as $\prod_{\rho=1}^{r \infty} X_{\rho}^{n_{\rho}}$, where the $X_{\rho}$ are subspaces which are pairwise not isometrically isomorphic and have trivial $\operatorname{Mult}\left(X_{\rho}\right)$; such a representation is always possible and is-up to rearrangement-unique (cf. [6]); then $X$ has the isomorphic Banach-Stone property

$$
\text { iff } \min n_{\rho}=1 \text {, and }
$$

$X$ has the strong isomorphic Banach-Stone property

$$
\text { iff } r=1 \text { and } n_{1}=1
$$

(which, by [3], is the same characterization as in the isometrical theory).

Another application of the results of $\S 4$ is discussed in $\S 6$ : how close can $\tau$-isomorphisms on a space be approximated by isometries?

1.8. Definition. We say that a Banach-space $X$ has the $A I$-property (which stands for "approximation by isometries") if for every $\varepsilon>0$ there is a $\tau>0$ such that every $\tau$-isomorphism lies in $\left(\operatorname{Iso}_{0}(X)\right)_{\varepsilon}$ (where Iso $_{0}(X)$ is the group of all isometries).

We prove that, for certain $X$, all $C(K, X)$ for arbitrary compact Hausdorff spaces $K$ have the AI-property. These results seem to be new even in the case $X=\mathbf{K}$ (where, however, there is some overlap with the work of Benyamini [8]; cf. also theorem 6.1 in Jarosz [17]). 
Some of our investigations, particularly in $\S 2$ and $\S 4$, are rather technical. It is suggested to the reader to consider first the special case $r=s=1$ and the case of compact instead of locally compact spaces.

Notation. Banach spaces are always real or complex and not reduced to zero, operators are linear and continuous. Topological spaces

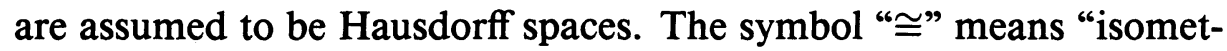
rically isomorphic" when applied to Banach spaces and "homeomorphic" in the case of topological spaces. Isomorphisms are always onto isomorphisms. The spaces $C_{0}(K, \mathbf{K})$ are abbreviated by $C_{0} K$ for locally compact $K$ and $C K$ for compact spaces. Finally, $B(x, r)$ stands for the closed ball with center $x$ and radius $r$ in a Banach space.

Acknowledgment. The author wishes to express his gratitude to Michael Cambern and his colleagues from the University of California at Santa Barbara for their hospitality during the author's stay in the summer of 1986 .

2. The constant $\alpha\left(X_{1}, \ldots, X_{n}\right)$. Let $T: C_{0}\left(K_{1}, \ldots, K_{r} ; X_{1}, \ldots, X_{r}\right) \rightarrow$ $C_{0}\left(L_{1}, \ldots, L_{s} ; Y_{1}, \ldots, Y_{s}\right)$ be a $\tau$-isomorphism. We will abbreviate

$$
\begin{aligned}
V & :=C_{0}\left(K_{1}, \ldots, K_{r} ; X_{1}, \ldots, X_{r}\right), \\
W & :=C_{0}\left(L_{1}, \ldots, L_{s} ; Y_{1}, \ldots, Y_{s}\right), \\
K & :=K_{1} \dot{\cup} \ldots \dot{\cup} K_{r}, \\
L & :=L_{1} \dot{\cup} \ldots \dot{\cup} L_{s},
\end{aligned}
$$

and we will associate certain points of $K$ with points of $L$.

2.1. Definition. Let $\alpha \geq 0$.

(i) For $k \in K$ and $l \in L$ we write $k \underset{T}{\stackrel{\alpha}{\rightarrow}} l$ if $\|(T f)(l)\| \leq \alpha\|f\|$ for every $f \in V$ such that $f(k)=0$.

(ii) $K \underset{T}{\stackrel{\alpha}{\rightarrow}} L$ means that for every $k$ there is an $l$ with $k \underset{T}{\stackrel{\alpha}{\rightarrow}} l$.

\subsection{Proposition.}

(i) Suppose that $k \underset{T}{\stackrel{\alpha}{\rightarrow}} l$. Then

$$
\|(T f)(l)\| \leq \alpha\|f\|+(1+\tau)\|f(k)\|
$$

for every $f \in V$.

(ii) Suppose that $k \underset{T}{\stackrel{\alpha}{\rightarrow}} l$ and $l \underset{T^{-1}}{\stackrel{\beta}{k}} \tilde{k}$. Then $k=\tilde{k}$ provided that $(\alpha+\beta)(1+\tau)<1$. Similarly $l \underset{T^{-1}}{\stackrel{\beta}{\rightarrow}} k, k \underset{T}{\stackrel{\alpha}{\rightarrow}} \tilde{l}$ yield $l=\tilde{l}$ under the same condition. 
(iii) Suppose that $K \underset{T}{\stackrel{\alpha}{\rightarrow}} L$ and $L \underset{T-1}{\stackrel{\beta}{\rightarrow}} K$ and that $(\alpha+\beta)(1+\tau)<1$. Then for every $k$ (resp. $l$ ) there is precisely one $l$ (resp. $k$ ) such that $k \underset{T}{\stackrel{\alpha}{\rightarrow}} l$ (resp. $l \underset{T^{-1}}{\stackrel{\beta}{\rightarrow}} k$ ). This induces mappings $\varphi: K \rightarrow L$ and $\psi: L \rightarrow$ $K$.

(iv) Under the conditions of (iii) we have $\varphi=\psi^{-1}$, and $\varphi$ and $\psi$ are continuous (i.e. $K \cong L$ ).

\section{Proof.}

(i) Let $f \in V$ be given and $\eta>0$. We choose $\tilde{f} \in V$ with $\|f\|=$ $\|\tilde{f}\|,\|f-\tilde{f}\| \leq\|f(k)\|+\eta, \tilde{f}(k)=0$. It follows that $\|(T \tilde{f})(l)\| \leq \alpha\|f\|$ and $\|T f-T \tilde{f}\| \leq(1+\tau)(\|f(k)\|+\eta)$ so that

$$
\|(T f)(l)\| \leq \alpha\|f\|+(1+\tau)(\|f(k)\|+\eta) .
$$

This proves the assertion since $\eta$ was arbitrary.

(ii) Suppose that $k \neq \tilde{k}$. We choose $f \in V$ with $\|f\|=1, f(k)=0$, $\|f(\tilde{k})\|=1$. By $k \underset{T}{\stackrel{\alpha}{\rightarrow}} l$ it follows that $\|(T f)(l)\| \leq \alpha$, and (i) yields

$$
\begin{aligned}
1 & =\|f(\tilde{k})\|=\left\|T^{-1}(T f)(\tilde{k})\right\| \\
& \leq \beta\|T f\|+(1+\tau)\|T f(l)\| \\
& \leq \beta(1+\tau)+(1+\tau) \alpha,
\end{aligned}
$$

a contradiction.

(iii) Let $k, l_{1}, l_{2}$ be given with $k \underset{T}{\stackrel{\alpha}{\rightarrow}} l_{1}, k \underset{T}{\stackrel{\alpha}{\rightarrow}} l_{2}$, and $l_{1} \neq l_{2}$. Choose $g \in W$ with $\|g\|=1, g\left(l_{1}\right)=0,\left\|g\left(l_{2}\right)\right\|=1$. (ii) yields $\left\|\left(T^{-1} g\right)(k)\right\| \leq$ $\beta$ so that, also by (ii),

$$
\begin{aligned}
1 & =\left\|g\left(l_{2}\right)\right\|=\left\|T\left(T^{-1} g\right)\left(l_{2}\right)\right\| \\
& \leq \alpha\left\|T^{-1} g\right\|+(1+\tau)\left\|T^{-1} g(k)\right\| \\
& \leq(\alpha+\beta)(1+\tau)
\end{aligned}
$$

which contradicts our assumption.

(iv) The assertion $\varphi=\psi^{-1}$ is a reformulation of (ii). Now let $k_{0} \in K$ be given and $U$ a neighbourhood of $\varphi\left(k_{0}\right)$. Choose a $g \in W$ with $\|g\|=\left\|g\left(\varphi\left(k_{0}\right)\right)\right\|=1, g(l)=0$ for $l \notin U$, and consider

$$
\tilde{U}:=\left\{k \mid\left\|\left(T^{-1} g\right)(k)\right\|>\beta\right\} .
$$

$l \notin U$ yields $\left\|\left(T^{-1} g\right)\left(\varphi^{-1}(k)\right)\right\| \leq \beta$ by definition so that $\varphi(\tilde{U}) \subset U$. 
We also have $k_{0} \in \tilde{U}$ since otherwise

$$
\begin{aligned}
1 & =\left\|g\left(l_{0}\right)\right\|=\left\|T\left(T^{-1} g\right)\left(l_{0}\right)\right\| \leq \alpha\left\|T^{-1} g\right\|+(1+\tau) \beta \\
& \leq(\alpha+\beta)(1+\tau) .
\end{aligned}
$$

This proposition motivates the following

2.3. Definition. Let $Y_{1}, \ldots, Y_{s}$ be Banach spaces. By $\alpha\left(Y_{1}, \ldots, Y_{s}\right)$ we mean the infimum of the numbers $\alpha>0$ with the following property: There exists a $\tau>0$ such that $K \underset{T}{\stackrel{\alpha}{\rightarrow}} L$ for all Banach spaces $X_{1}, \ldots, X_{r}$, all locally compact spaces $K_{1}, \ldots, K_{r}, L_{1}, \ldots, L_{s}$ and all $\tau$ isomorphism $T: V \rightarrow W$ (notation as above).

As an immediate reformulation of 2.2 we then have

2.4. Theorem. Suppose that $\alpha\left(X_{1}, \ldots, X_{r}\right)+\alpha\left(Y_{1}, \ldots, Y_{s}\right)<1$. Then there exists a $\tau>0$ such that, whenever there is a $\tau$-isomorphism

$$
T: C_{0}\left(K_{1}, \ldots, K_{r} ; X_{1}, \ldots, X_{r}\right) \rightarrow C_{0}\left(L_{1}, \ldots, L_{s} ; Y_{1}, \ldots, Y_{s}\right),
$$

then necessarily $K_{1} \dot{\cup} \cdots \dot{\cup} K_{r} \cong L_{1} \dot{\cup} \cdots \dot{\cup} L_{s}$.

Proof. Choose $\alpha>\alpha\left(X_{1}, \ldots, X_{r}\right), \beta>\alpha\left(Y_{1}, \ldots, Y_{s}\right)$ such that $\alpha+\beta<$ 1. By definition there is a $\tau>0$ such that the existence of $T: V \rightarrow W$ yields $K \underset{T}{\stackrel{\alpha}{\rightarrow}} L$ and $L \underset{T-1}{\stackrel{\beta}{\rightarrow}} K$. Without loss of generality we may assume that $(\alpha+\beta)(1+\tau)<1$ so that Proposition 2.2 applies.

We now are going to investigate further properties of the mapping $\varphi$ of 2.2 .

Let $T$ be given as in 2.2 , suppose that $(\alpha+\beta)(1+\tau)<1$, and apply 2.2 (iii) to obtain $\varphi$.

Let $k \in K$ be arbitrarily given, say $k \in K_{\rho}$ and $\varphi(k)=: l \in L_{\sigma}$. We choose a fixed $h \in C_{0} K$ with support in $K_{\rho}$ and $\|h\|=h(k)=1$ and define

$$
S_{k}: X_{\rho} \rightarrow Y_{\sigma}
$$

by $S_{k}(x):=\left(T x^{\sim}\right)(l)$, where $x^{\sim}$ denotes the function

$$
x^{\sim}(k):=\left\{\begin{aligned}
h(k) x & \text { for } k \in K_{\rho}, \\
0 & \text { otherwise. }
\end{aligned}\right.
$$

Clearly $S_{k}$ is an operator with $\left\|S_{k}\right\| \leq 1+\tau$.

Similarly we define $R_{l}: Y_{\sigma} \rightarrow X_{\rho}$, this time using a fixed $\hat{h} \in C_{0} L$ and $T^{-1}$ instead of $T$. Fix an $x \in X_{\rho}$. We have

$$
\left[T\left(x^{\sim}\right)-S_{k}(x)^{\sim}\right](l)=0
$$


so that

$$
\left\|T^{-1}\left[T\left(x^{\sim}\right)-S_{k}(x)^{\sim}\right](k)\right\| \leq \beta\left\|T x^{\sim}-S_{k}(x)^{\sim}\right\| \leq 2(1+\tau) \beta\|x\| .
$$

But this means just

$$
\left\|x-R_{l} S_{k} x\right\| \leq 2(1+\tau) \beta\|x\|,
$$

and similarly $\left\|y-S_{k} R_{l} y\right\| \leq 2(1+\tau) \alpha\|y\|$ is valid. It is then a simple exercise to show that this implies that $S_{k}$ is an isomorphism with $\left\|S_{k}\right\|\left\|S_{k}^{-1}\right\| \leq \hat{\alpha}:=(1+\tau)^{2} \min \left\{(1-2(1+\tau) \alpha)^{-1},(1-2(1+\tau) \beta)^{-1}\right\}$, provided that $\alpha$ and $\beta$ are smaller than $1 /[2(1+\tau)]$. Thus $d\left(X_{\rho}, Y_{\sigma}\right) \leq$ $\hat{\alpha}$, and consequently $d\left(Y_{\sigma}, Y_{\sigma^{\prime}}\right) \leq \hat{\alpha}^{2}$ if $k, k^{\prime} \in K_{\rho}$ are given such that $\varphi(k) \in L_{\sigma}, \varphi\left(k^{\prime}\right) \in L_{\sigma^{\prime}}$. In particular, if the $d\left(Y_{\sigma}, Y_{\sigma^{\prime}}\right)$ are all greater than $\hat{\alpha}^{2}$, then all $k \in K_{\rho}$ are mapped to the same $L_{\sigma}$, i.e. $\varphi\left(K_{\rho}\right) \subset L_{\sigma}$. Considering $\varphi^{-1}$ we get $\varphi^{-1}\left(L_{\sigma}\right) \subset K_{\rho}$, so that $\varphi$ induces a homeomorphism between $K_{\rho}$ and $L_{\sigma}$. Summing up, we have proved the

2.5. Theorem. Suppose that

$$
\alpha_{X}:=\alpha\left(X_{1}, \ldots, X_{r}\right), \quad \alpha_{Y}:=\alpha\left(Y_{1}, \ldots, Y_{l}\right)<\frac{1}{2}
$$

and that

$$
d\left(X_{\rho}, X_{\rho^{\prime}}\right), d\left(Y_{\sigma}, Y_{\sigma^{\prime}}\right)>\min \left\{\left(1-2 \alpha_{X}\right)^{-2},\left(1-2 \alpha_{Y}\right)^{-2}\right\}=: \hat{\alpha}_{0}^{2} .
$$

Then for every $\varepsilon>0$ there is a $\tau>0$ such that the existence of a $\tau$-isomorphism $T: V \rightarrow W$ implies that $r=s$ and that there exists a bijection $\omega:\{1, \ldots, r\} \rightarrow\{1, \ldots, s\}$ such that $K_{\rho} \cong L_{\omega(\rho)}$ and $d\left(X_{\rho}, Y_{\omega(\rho)}\right) \leq \hat{\alpha}_{0}+\varepsilon$ for every $\rho$.

Proof. Choose $\alpha>\alpha_{X}, \beta>\beta_{Y}, \tau>0$ such that the above considerations apply (with $\hat{\alpha}=\hat{\alpha}_{0}+\varepsilon$ ).

3. Operators which are nearly multipliers and the constant $\alpha_{2}(X)$. In this section we discuss the class $\operatorname{Mult}_{\varepsilon}(X)$ (see Def. 1.6(ii)). We determine $\alpha_{2}(X)$ for some classes of spaces and prepare the proof of our main results in $\S 4$. The following assertions are immediate:

\subsection{LEMMA.}

(i) $\operatorname{Mult}_{\varepsilon}(X)$ is a closed subset of the ball with radius $1+\varepsilon$.

(ii) $\bigcap_{\varepsilon>\varepsilon_{0}} \operatorname{Mult}_{\varepsilon}(X)=\operatorname{Mult}_{\varepsilon_{0}}(X)$ for every $\varepsilon_{0} \geq 0$, so that in particular $\bigcap_{\varepsilon>0} \operatorname{Mult}_{\varepsilon}(X) \subset \operatorname{Mult}(X)$. 
We start by investigating two classes of examples.

3.2. Theorem. Let $X$ be finite-dimensional.

(i) For every $\alpha>0$ there is an $\varepsilon>0$ such that

$$
\operatorname{Mult}_{\varepsilon}(X) \subset(\operatorname{Mult}(X))_{\alpha} .
$$

(ii) Suppose that in addition $\operatorname{Mult}(X)$ is trivial. Then $\alpha_{2}(X)=0$.

Proof. (i) follows from a simple compactness argument by using the compactness of $\{R \mid R \in \operatorname{Mult}(X),\|R\| \leq 1\}$ and 3.1(ii). (ii) is a consequence of (i).

3.3. THEOREM. Let $X$ be uniformly smooth; this means that for every $\eta>0$ there is an $\tilde{\varepsilon}>0$ such that $\|x-y\| \leq \tilde{\varepsilon}$ implies that $\|x+y\| \geq\|x\|+\|y\|-\eta\|x-y\|$ (see [13], p. 147). Then $\alpha_{2}(X)=0$.

Proof. The proof is sketched in several steps. Details are left to the reader

1. For every two-dimensional Banach space $X_{0}$ there exist $x_{0}, y_{0} \in$ $X_{0}$ with norm one such that

$$
\{\tilde{x} \mid\|\tilde{x}\| \leq 1\} \subset\left\{\alpha x_{0}+\beta y_{0}|| \alpha|,| \beta \mid \leq 1\right\}
$$

(choose $x_{0}, y_{0}$ to be an Auerbach-system; see 1.c.3 in [16]).

2. Let $R: X \rightarrow X$ be an operator on any Banach space $X$ such that, for a fixed $\varepsilon \geq 0$, we have $\left\|R x-\lambda_{x} x\right\| \leq \varepsilon$ for each $x$ in the unit ball, where $\lambda_{x} \in \mathbf{K}$ may depend on $x$ (i.e. every $x$ is nearly an eigenvector). Then there is a $\lambda \in \mathbf{K}$ such that $\left\|R-\lambda \operatorname{Id}_{X}\right\| \leq 18 \varepsilon$. (First let $x, y$ be vectors as in " 1 .". With $\lambda_{x}, \lambda_{y}, \lambda \in \mathbf{K}$ such that

$$
\left\|R x-\lambda_{x} x\right\|, \quad\left\|R y-\lambda_{y} y\right\|, \quad\left\|R\left(\frac{x+y}{\|x+y\|}\right)-\lambda\left(\frac{x+y}{\|x+y\|}\right)\right\| \leq \varepsilon
$$

it follows that $\left\|\left(\lambda_{x}-\lambda\right) x+\left(\lambda_{y}-\lambda\right) y\right\| \leq 4 \varepsilon$ so that $\left|\lambda_{x}-\lambda_{y}\right| \leq 8 \varepsilon$.)

Now let $x, y$ be arbitrary with $\|x\|=\|y\|=1$. We choose $\lambda_{x}, \lambda_{y}$ with $\left\|R x-\lambda_{x} x\right\|,\left\|R y-\lambda_{y} y\right\| \leq \varepsilon$, and we claim that $\left|\lambda_{x}-\lambda_{y}\right| \leq 18 \varepsilon$.

To this end, let $x_{0}, y_{0} \in \operatorname{lin}\{x, y\}$ be as in "1." (we note that without loss of generality $x$ and $y$ are linearly independent). By the first part of the proof we know that $\left|\lambda_{x_{0}}-\lambda_{y_{0}}\right| \leq 8 \varepsilon$ is true for the associated $\lambda$ 's. Therefore the numbers $\left\|\left(R-\lambda_{x_{0}} \operatorname{Id}_{X}\right)(z)\right\|$ are bounded by $8 \varepsilon$ for $z$ in $\left\{\alpha x_{0}+\beta y_{0}|| \alpha|,| \beta \mid \leq 1\right\}$, and in particular we have

$$
\left\|R x-\lambda_{x_{0}} x\right\|, \quad\left\|R y-\lambda_{x_{0}} y\right\| \leq 8 \varepsilon
$$

so that $\left|\lambda_{x}-\lambda_{y}\right| \leq 18 \varepsilon$. 
Now choose any $x_{0}$ with $\left\|x_{0}\right\|=1$ and define $\lambda:=\lambda_{x_{0}}$. Then obviously $\left\|R-\lambda \operatorname{Id}_{X}\right\| \leq 18 \varepsilon$.)

3. Let $\varepsilon \geq 0$. For any $x$ we define

$$
I(x, \varepsilon ; X):=\bigcap B\left(x_{0}, r(1+\varepsilon)\right),
$$

where the intersection runs over all $x_{0} \in X, r \geq 0$ such that $\Theta x \in$ $B\left(x_{0}, r\right)$ for every $\Theta$ with $|\Theta|=1$. Then clearly $R \in \operatorname{Mult}_{\varepsilon}(X)$ iff $R x \in I(x, \varepsilon ; X)$ for every $x$ with $\|x\| \leq 1$.

4. Let $X$ be a Banach space. Suppose that for every $\alpha>0$ there is an $\varepsilon>0$ such that

$$
I(x, \varepsilon ; X) \subset(\mathbf{K} x)_{\alpha}
$$

for any $x$ with $\|x\| \leq 1$. Then $\alpha_{2}(X)=0$ (as an immediate consequence of " 2 ." and " 3 .").

5. Let $Y$ be a two-dimensional smooth Banach space. Then for every $\alpha>0$ there is an $\varepsilon>0$ with

$$
I(x, \varepsilon ; Y) \subset(\mathbf{K} x)_{\alpha} \quad(\text { all } x \text { with }\|x\| \leq 1) .
$$

This follows from a compactness argument and the fact that $I(x, 0 ; Y)$ $\subset \mathbf{K} x$ for smooth spaces.

6. Let $\tilde{\varepsilon}:] 0,1] \rightarrow] 0,1]$ be a fixed function. In $\mathscr{B}$, the set of all two-dimensional Banach spaces provided with the distance $\log d(\cdot, \cdot)$, we consider the subset $\mathscr{B}_{\tilde{\varepsilon}}: Y \in \mathscr{B}_{\tilde{\varepsilon}}$ iff

$$
\begin{array}{r}
\|x-y\| \leq \tilde{\varepsilon}(\eta) \Rightarrow\|x+y\| \geq\|x\|+\|y\|-\eta\|x-y\| \\
\quad(\text { all } \eta \in] 0,1], x, y \in Y) .
\end{array}
$$

Then $\mathscr{B}_{\tilde{\varepsilon}}$ is closed in $\mathscr{B}$ and thus compact.

7. Let $\tilde{\varepsilon}$ be as in "6.". Then for every $\alpha>0$ there is an $\varepsilon>0$ such that

$$
I(x, \varepsilon ; Y) \subset(\mathbf{K} x)_{\alpha}
$$

uniformly for all $Y$ in $\mathscr{B}_{\tilde{\varepsilon}}$ and $x \in Y$ with $\|x\| \leq 1$ (this follows from "5." and "6." and a continuity argument).

8. Now let $X$ be a uniformly smooth Banach space. Then there is, by definition, a function $\tilde{\varepsilon}:] 0,1] \rightarrow$ ]0, 1] such that all two-dimensional subspaces of $X$ belong to $\mathscr{B}_{\tilde{\varepsilon}}$. Let $\alpha>0$ be given and $\varepsilon$ as in "7.". Then, for $x \in X$ with $\|x\| \leq 1$, we have

$$
I(x, \varepsilon ; Y) \subset(\mathbf{K} x)_{\alpha}
$$

for every two-dimensional subspace which contains $x$. But this implies $I(x, \varepsilon ; X) \subset(\mathbf{K} x)_{\alpha}$.

Now a combination of " $8 . "$ and " 4 ." proves the theorem. 
We return to the spaces

$$
C\left(K_{1}, \ldots, K_{r} ; X_{1}, \ldots, X_{r}\right), \quad C\left(L_{1}, \ldots, L_{s} ; Y_{1}, \ldots, Y_{s}\right),
$$

and we adopt the notation of $\S 2$. Since for every bounded and continuous $h: K \rightarrow \mathbf{K}$ with $\|h\| \leq 1$ the operator $M_{h}: V \rightarrow V$, defined by $f \mapsto h f$, lies in $\operatorname{Mult}_{0}(V)$, the operator $T M_{h} T^{-1}$ lies in $\operatorname{Mult}_{2 \tau+\tau^{2}}(W)$ for every $\tau$-isomorphism $T$. The proof of these facts is straightforward.

This observation which will be of crucial importance later necessitates the study of the $\operatorname{Mult}_{\varepsilon}(V), \operatorname{Mult}_{\varepsilon}(W)$.

3.4. Proposition. Let $\varepsilon \geq 0$ and $R \in \operatorname{Mult}_{\varepsilon}(V)$.

(i) Fix $\rho \in\{1, \ldots, r\}$ and $k \in K_{\rho}$. With $x^{\sim}$ as in $\S 2$ (cf. the discussion leading to Th. 2.5) we define $R_{k}: X_{\rho} \rightarrow X_{\rho}$ by $x \mapsto\left(R x^{\sim}\right)(k)$. Then $R_{k} \in \operatorname{Mult}_{\varepsilon}\left(X_{\rho}\right)$.

(ii) For $f \in V$ with $f(k)=0$ we have $\|(R f)(k)\| \leq \varepsilon\|f\|$.

(iii) For every $f$ and every $k$ we have

$$
\left\|R_{k} f(k)\right\|-2 \varepsilon\|f\| \leq\|(R f)(k)\| \leq\left\|R_{k} f(k)\right\|+2 \varepsilon\|f\| .
$$

Proof.

(i) $\left\|\Theta x-x_{0}\right\| \leq 1$ (all $|\Theta|=1$ ) yields $\left\|\Theta x^{\sim}-x_{0}^{\sim}\right\| \leq 1$ and thus $\left\|R x^{\sim}-x_{0}^{\sim}\right\| \leq 1+\varepsilon$. Evaluating this at $k$ gives $\left\|R_{k} x-x_{0}\right\| \leq 1+\varepsilon$.

(ii) Let $x \in X_{\rho}$ with $\|x\|=1$ be given. For $\eta>0$ we choose an $h \in C_{0} K$ with support in $K_{\rho} \cap\{\|f\|<\eta\}$ such that $h(k)=\|h\|=1-\eta$ (where $f \in V, f(k)=0,\|f\| \leq 1$ ). Define $h x$ by

$$
(h x)\left(k^{\prime}\right)=\left\{\begin{aligned}
h\left(k^{\prime}\right) x & \text { if } k^{\prime} \in K_{\rho} \\
0 & \text { otherwise. }
\end{aligned}\right.
$$

Then $\|\Theta f-h x\| \leq 1$ for every $\Theta$ with $|\Theta|=1$ so that $\|R f-h x\| \leq$ $1+\varepsilon$. The special choice $x:=(R f)(k) /\|R f(k)\|$ gives, by considering $(R f-h x)(k)$,

$$
1-\eta+\|R f(k)\| \leq 1+\varepsilon
$$

This proves that $\|R f(k)\| \leq \varepsilon$ since $\eta>0$ was arbitrary.

(iii) Let $f \in V$ and $k \in K$ be given. $f-f(k)^{\sim}$ vanishes at $k$ so that by (ii) $\left\|(R f)(k)-R_{k} f(k)\right\| \leq 2 \varepsilon\|f\|$.

Using this preparation we can prove that property of the $R \in$ $\operatorname{Mult}_{\varepsilon}(X)$ which will be very important in $\S 4$. 
3.5. Corollary. Let $\alpha, \eta, \varepsilon>0$ be given and $k \in K$. We assume that

(i) $R \in \operatorname{Mult}_{\varepsilon}(V)$,

(ii) there is a $g_{0} \in V$ such that $\left\|g_{0}\right\|=1,\left\|R g_{0}(k)\right\| \geq 1-\eta$,

(iii) $\operatorname{Mult}_{\varepsilon}\left(X_{\rho}\right) \subset\left(\mathbf{K} \operatorname{Id}_{X_{\rho}}\right)_{\alpha}$.

Then $\|(R f)(k)\| \geq\|f(k)\|-(2 \alpha+4 \varepsilon+\eta)\|f\|$ for every $f \in V$ (roughly: with $f$ also $R f$ is large at $k$, and this can be asserted at every $k$ where $R g_{0}$ is large for at least one $g_{0}$ ).

Proof. 3.4(iii), applied for $g_{0}$, gives $\left\|R_{k}\right\| \geq 1-\eta-2 \varepsilon$. Since $R_{k} \in \operatorname{Mult}_{\varepsilon}\left(X_{\rho}\right) \subset\left(\mathbf{K I d}_{X_{\rho}}\right)_{\alpha}$ we find a $\lambda \in \mathbf{K}$ with $\left\|R_{k}-\lambda \operatorname{Id}_{X_{\rho}}\right\| \leq \alpha$. Here necessarily $|\lambda| \geq 1-\eta-2 \varepsilon-\alpha$. Now let $f \in V$ be given. Again by 3.4 (iii) we have

$$
\begin{aligned}
\|(R f)(k)\| & \geq\left\|R_{k} f(k)\right\|-2 \varepsilon\|f\| \\
& \geq\|\lambda f(k)\|-2 \varepsilon\|f\|-\alpha\|f(k)\| \\
& \geq\|f(k)\|-(2 \alpha+4 \varepsilon+\eta)\|f\| .
\end{aligned}
$$

4. An estimate of the constant $\alpha\left(X_{1}, \ldots, X_{r}\right)$ and some important consequences. The following theorem is doubtlessly the most important one in this paper. Only by using this we are able to apply the results of $\S 2$ (by having in mind, of course, our knowledge about the $\alpha_{1}(X)$, $\left.\alpha_{2}(X)\right)$.

\subsection{ThEOREM. $\alpha\left(X_{1}, \ldots, X_{r}\right) \leq \max _{\rho} \min \left\{\alpha_{1}\left(X_{\rho}\right), 2 \alpha_{2}\left(X_{\rho}\right)\right\}$.}

Proof. For the sake of convenience we prove the theorem for a family $Y_{1}, \ldots, Y_{s}$ (which avoids the change of notation).

Let $\alpha>\max _{\sigma} \min \left\{\alpha_{1}\left(Y_{\sigma}\right), 2 \alpha_{2}\left(Y_{\sigma}\right)\right\}$ arbitrary. We assume that the $Y_{\sigma}$ are given such that

$$
\begin{aligned}
& \alpha>\alpha_{1}\left(Y_{\sigma}\right) \quad \text { for } \sigma=1, \ldots, \hat{s}, \quad \text { and } \\
& \alpha>2 \alpha_{2}\left(Y_{\sigma}\right) \text { for } \sigma=\hat{s}+1, \ldots, s .
\end{aligned}
$$

It has to be shown that there is a $\tau>0$ such that $K \underset{T}{\stackrel{\alpha}{\rightarrow}} L$ whenever $X_{1}, \ldots, X_{r}, K_{1}, \ldots, K_{r}, L_{1}, \ldots, L_{s}$ are given and there exists a $\tau$ isomorphism $T: V \rightarrow W$ (notation as in $\S 2$ ).

At first we choose any $\alpha^{\prime}$, $\alpha^{\prime \prime}$ with $\alpha_{2}\left(Y_{\sigma}\right)<\alpha^{\prime}<\alpha^{\prime \prime}<\alpha / 2$ for $\sigma=\hat{s}+1, \ldots, s$ and $\alpha_{1}\left(Y_{\sigma}\right)<2 \alpha^{\prime \prime}$ for $\sigma=1, \ldots, \hat{s}$, and then an $\varepsilon>0$ with

$$
-2 \alpha^{\prime}+4 \varepsilon<2 \alpha^{\prime \prime}
$$


$-1-\varepsilon \leq\left\|x_{0}\right\| \leq 1+\varepsilon,\left\|x_{0}+\Theta x\right\| \leq 1+\varepsilon$ for all $|\Theta|=1$ implies $\|x\| \leq 2 \alpha^{\prime \prime}$; all $x_{0}, x \in Y_{\sigma}$, where $\sigma=1, \ldots, \hat{s}$,

- $\operatorname{Mult}_{\varepsilon}\left(Y_{\sigma}\right) \subset\left(\mathbf{K} \operatorname{Id}_{Y_{\sigma}}\right)_{\alpha^{\prime}}$ for $\sigma=\hat{s}+1, \ldots, s$.

Finally we suppose that $\tau>0$ is so small that

$$
\begin{aligned}
& (1+\tau)^{2} \leq 1+\varepsilon, \\
- & 1-2 \tau-\tau^{2} /(1+\tau) \geq 1-\varepsilon, \\
- & \left(2 \alpha^{\prime}+4 \varepsilon+\eta_{\tau}+\tau\right)(1+\tau) \leq 2 \alpha^{\prime \prime} \\
& \text { where } \eta_{\tau}:=\left(1-2 \tau-\tau^{2}\right) /(1+\tau)^{2}-1 .
\end{aligned}
$$

Now let $X_{1}, \ldots, X_{r}, K_{1}, \ldots, K_{r}, L_{1}, \ldots, L_{s}$ and a $\tau$-isomorphism $T$ : $V \rightarrow W$ be given. We claim that $K \underset{T}{\stackrel{\alpha}{\rightarrow}} L$, i.e.

$$
\alpha_{k}:=\inf _{l} \sup \{\|(T f)(l)\| \mid f \in V, f(k)=0,\|f\| \leq 1\}<\alpha
$$

for every $k \in K$. We will show that $t<\alpha_{k}$ implies $t \leq 2 \alpha^{\prime \prime}$ so that $\alpha_{k} \leq 2 \alpha^{\prime \prime}<\alpha$. Let $k$ and such a $t$ be given and fix any function $f_{0} \in V$ with $\left\|f_{0}\right\|=\left\|f_{0}(k)\right\|=1$. The set $\tilde{L}_{\tau}:=\left\{l \mid\left\|T f_{0}(l)\right\| \geq \tau\right\}$ is compact so that we find $f_{1}, \ldots, f_{n} \in V$ with $\left\|f_{i}\right\| \leq 1, f_{i}(k)=0$, $\max _{i}\left\|T f_{i}(l)\right\|>t$ for every $l \in \tilde{L}_{\tau}$.

Since all these $f_{i}$ vanish at $k_{0}$ it is possible to choose an $h \in C_{0} K$ with $\left\|h f_{i}\right\| \leq \tau,\|h\|=-h(k)=1,\left\|\Theta f_{i}-h f_{0}\right\| \leq 1+\tau$ for $i=1, \ldots, n$ and $|\Theta|=1$.

We have $\left\|f_{0}-h f_{0}\right\|=2$ so that there is an $l_{0} \in L$ with $\|\left[T f_{0}-\right.$ $\left.T\left(h f_{0}\right)\right]\left(l_{0}\right) \| \geq 2 /(1+\tau)$ which yields

$$
\left\|\left[T\left(h f_{0}\right)\right]\left(l_{0}\right)\right\|, \quad\left\|\left(T f_{0}\right)\left(l_{0}\right)\right\| \geq\left(1-2 \tau-\tau^{2}\right) /(1+\tau)
$$

(note that $\left.\left\|T\left(h f_{0}\right)\right\|,\left\|T f_{0}\right\| \leq 1+\tau\right)$. Therefore in particular $l_{0} \in \tilde{L}_{\tau}$, and we find an $i_{0} \in\{1, \ldots, n\}$ with $\left\|\left(T f_{i_{0}}\right)\left(l_{0}\right)\right\|>t$. $l_{0}$ lies in one of the $L_{\sigma}$, and we consider two cases.

Case 1. $\sigma \in\{1, \ldots, \hat{s}\}$.

We know that $\left\|\Theta f_{i_{0}}-h f_{0}\right\| \leq 1+\tau$ for all $|\Theta|=1$, i.e. with $x_{0}:=$ $\left[T\left(h f_{0}\right)\right]\left(l_{0}\right), x:=\left(T f_{i_{0}}\right)\left(l_{0}\right)$ we have

$$
\begin{aligned}
\left(1-2 \tau-\tau^{2}\right) /(1+\tau) & \leq\left\|x_{0}\right\| \leq(1+\tau)^{2} \\
t & <\|x\| \\
\left\|\Theta x-x_{0}\right\| & \leq(1+\tau)^{2} \quad(\text { all }|\Theta|=1) .
\end{aligned}
$$

By the choice of $\tau$ this implies $\|x\| \leq 2 \alpha^{\prime \prime}$, and thus $t \leq 2 \alpha^{\prime \prime}$ as claimed.

Case 2. $\sigma \in\{\hat{s}+1, \ldots, s\}$.

The operator $M_{h}: V \rightarrow V, f \mapsto h f$, belongs to $\operatorname{Mult}_{0}(V)$ so that $R:=T M_{h} T^{-1}$ lies in $\operatorname{Mult}_{2 \tau+\tau^{2}}(W) \subset \operatorname{Mult}_{\varepsilon}(W)$. 
With $g_{0}:=T f_{0} /\left\|T f_{0}\right\|$ we have $R g_{0}=T\left(h f_{0}\right) /\left\|T f_{0}\right\|$ and consequently $\left\|\left(R g_{0}\right)\left(l_{0}\right)\right\| \geq\left(1-2 \tau-\tau^{2}\right) /(1+\tau)^{2}=1-\eta_{\tau}$. On the other hand we know that $\left\|h f_{i_{0}}\right\| \leq \tau$ so that $\left\|R\left(T f_{i_{0}}\right)\right\|=\left\|T\left(h f_{i_{0}}\right)\right\| \leq \tau(1+\tau)$.

Now Corollary 3.5 comes into play. Since $R \in \operatorname{Mult}_{\varepsilon}(W)$ and $\operatorname{Mult}_{\varepsilon}\left(Y_{\sigma}\right) \subset\left(\operatorname{KId}_{Y_{\sigma}}\right)_{\alpha^{\prime}}$ it follows that

$$
\begin{aligned}
t<\left\|T f_{i_{0}}\left(l_{0}\right)\right\| & \leq\left(2 \alpha^{\prime}+4 \varepsilon+\eta_{\tau}\right)\left\|T f_{i_{0}}\right\|+\left\|R\left(T f_{i_{0}}\right)\right\| \\
& \leq\left(2 \alpha^{\prime}+4 \varepsilon+\eta_{\tau}+\tau\right)(1+\tau) \leq 2 \alpha^{\prime \prime},
\end{aligned}
$$

and this completes the proof.

We now turn to some interesting consequences which can be derived very simply by combining Theorem 2.4 or Theorem 2.5 with Theorem 3.2, Theorem 3.3, the remarks following Definition 1.5 and, of course, Theorem 4.1.

4.2. Theorem. Suppose that $\min \left\{\alpha_{1}(X), 2 \alpha_{2}(X)\right\}<\frac{1}{2}$ for $X=$ $X_{\rho}(\rho=1, \ldots, r)$ and $X=Y_{\sigma}(\sigma=1, \ldots, s)$.

Then there is a $\tau>0$ such that the existence of a $\tau$-isomorphism

$$
T: C_{0}\left(K_{1}, \ldots, K_{r} ; X_{1}, \ldots, X_{r}\right) \rightarrow C_{0}\left(L_{1}, \ldots, L_{s} ; Y_{1}, \ldots, Y_{s}\right)
$$

implies that $K_{1} \dot{\cup} \cdots \dot{\cup} K_{r} \cong L_{1} \dot{\cup} \cdots \dot{U} L_{s}$.

4.3. TheOREM. Suppose that $\min \left\{\alpha_{1}(X), 2 \alpha_{2}(X)\right\}<\frac{1}{2}$. Then there is $a \delta>0$ such that

$$
d\left(C_{0}(K, X), C_{0}(L, X)\right) \geq 1+\delta
$$

for arbitrary $K, L$ with $K \not L$.

4.4. TheOREM. Suppose that $\alpha_{1}(X)=\alpha_{2}(X)=0$ for $X=X_{\rho}(\rho=$ $1, \ldots, r)$ and $X=Y_{\sigma}(\sigma=1, \ldots, s)$. Suppose further that $d\left(X_{\rho}, X_{\rho^{\prime}}\right)$, $d\left(Y_{\sigma}, Y_{\sigma^{\prime}}\right)>1$ for $\rho \neq \rho^{\prime}, \sigma \neq \sigma^{\prime}$. Then for every $\delta>0$ there exists $a$ $\tau>0$ such that the following holds: whenever

$$
T: C_{0}\left(K_{1}, \ldots, K_{r} ; X_{1}, \ldots, X_{r}\right) \rightarrow C_{0}\left(L_{1}, \ldots, L_{s} ; Y_{1}, \ldots, Y_{s}\right)
$$

is a $\tau$-isomorphism, then $r=s$, and there is a bijection $\omega:\{1, \ldots, r\} \rightarrow$ $\{1, \ldots, s\}$ with $K_{\rho} \cong L_{\omega(\rho)}$ and $d\left(X_{\rho}, Y_{\omega(\rho)}\right) \leq 1+\delta$ for every $\rho$.

Note. In particular this can be applied to such situations where for every $\tau>0 \tau$-isomorphisms exist. Then $d\left(X_{\rho}, Y_{\omega(\rho)}\right)=1$ for all $\rho$.

5. Isomorphic Banach-Stone theorems. We now state our main results on isomorphic Banach-Stone theorems. They appear as special cases of our more general approach. 
5.1. THEOREM. Each of the following conditions implies that $X$ has the isomorphic Banach-Stone property:

(i) $\min \left\{\alpha_{1}(X), 2 \alpha_{2}(X)\right\}<\frac{1}{2}$;

(ii) $X$ is of the form $\prod_{\rho=1}^{r \infty} X_{\rho}^{n_{\rho}}$, where $d\left(X_{\rho}, X_{\rho^{\prime}}\right) \geq 1+\delta_{0}$ for $a$ suitable $\delta_{0}>0$ and all $\rho \neq \rho^{\prime}$. Further $\min n_{\rho}=1$ and

$$
\max _{\rho} \min \left\{\alpha_{1}\left(X_{\rho}\right), 2 \alpha_{2}\left(X_{\rho}\right)\right\}<1-\left(1+\delta_{0}\right)^{-1 / 2} \approx \delta_{0} / 2 .
$$

Proof. One only has to note that $C_{0}\left(K, \prod X_{\rho}^{n_{\rho}}\right)$ can be identified with $C_{0}\left(n_{1} K, \ldots, n_{r} K ; X_{1}, \ldots, X_{r}\right)$; here $n K$ stands for the disjoint union of $n$ copies of $K$.

5.2. THEOREM. Each of the following conditions implies that $X$ has the strong isomorphic Banach-Stone property:

(i) $X$ is uniformly smooth;

(ii) $X$ is uniformly convex;

(iii) $X$ is finite-dimensional with $\operatorname{Mult}(X)=\mathbf{K} \mathrm{Id}_{X}$;

(iv) $\min \left\{\alpha_{1}(X), 2 \alpha_{2}(X)\right\}=0$;

(v) $\alpha(X)=0$.

Proof. In view of Theorem 3.3, the remark following Definition 1.5, Theorem 3.2(ii), and Theorem 4.1 it suffices to prove (v). Let $\varepsilon>0$ be given and $\alpha>0$ so small that $(1+\alpha)^{2} /(1-2 \alpha(1+\alpha)) \leq 1+\varepsilon$. Choose $\tau>0$ for this $\alpha$ according to 2.3, where $\tau \leq \alpha$. Now let any $\tau$ isomorphism $T: C_{0}(K, X) \rightarrow C_{0}(L, X)$ be given. With $\varphi$ and $\left(S_{k}\right)_{k \in K}$ as in the discussion leading to Theorem 2.5 we define $T_{k}:=S_{k}$.

The $T_{k}$ are isomorphisms, and

$$
\left\|T_{k}\right\|\left\|T_{k}^{-1}\right\| \leq(1+\tau)^{2} /(1-2(1+\tau) \alpha) \leq 1+\varepsilon .
$$

It remains to prove that $\left\|T f(\varphi(k))-T_{k} f(k)\right\| \leq \varepsilon$ for all $k$ and $f$ with $\|f\| \leq 1$. Let such $f$ and $k$ be given. With $\varphi(k)=: l$ we have

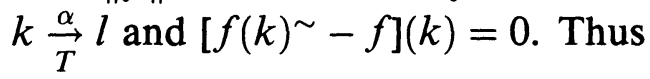

$$
\left\|T_{k}(f(k))-T f(\varphi(k))\right\|=\left\|T\left[f(k)^{\sim}-f\right](l)\right\| \leq 2 \alpha \leq \varepsilon
$$

which completes the proof.

5.3. THEOREM. Let $X$ be a Banach space which can be written as $X=\prod_{\rho=1}^{r} X_{\rho}^{n_{\rho}}$, where $d\left(X_{\rho}, X_{\rho^{\prime}}\right)>1$ for $\rho \neq \rho^{\prime}$ and $\alpha\left(X_{\rho}\right)=0$ for $\rho=$ $1, \ldots, r$. We emphasize that this is possible for every finite-dimensional space (combine Theorem 3.2 (ii) with Proposition 5.1 in [6]) but also surely for many other spaces. 
(i) $X$ has the isomorphic Banach-Stone property iff $\min n_{\rho}=1$.

(ii) $X$ has the strong isomorphic Banach-Stone property iff $r=n_{1}=$ 1.

Proof. (i) If $\min n_{\rho}=1$ then $X$ has the Banach-Stone property by Theorem 5.1. If $\min n_{\rho}>1$ then $X$ does not even have the usual Banach-Stone property since there are non-homeomorphic $K, L$ with $n K \cong n L$ for every $n \geq 2$ (so that for these $K, L$ we have $K \not L L$ but $\left.C_{0}(K, X) \cong C_{0}(L, X)\right)$; see [4], Corollary 11.15 .

(ii) One implication is proved in Theorem 5.2.

Conversely, if $r>1$ or $r=1$ and $n_{1}>1$ then $X$ can be written as $J \oplus_{\infty} J^{\perp}$ for suitable nontrivial subspaces $J, J^{\perp}\left(\oplus_{\infty}\right.$ denotes the $L^{\infty}$. direct sum). With $K=L=\{1,2\}$ we identify $C_{0}(K, X)$ and $C_{0}(L, X)$ with $J \oplus_{\infty} J \oplus_{\infty} J^{\perp} \oplus_{\infty} J^{\perp}$. An isometry $T$ on this space is defined by $\left(j_{1}, j_{2}, j_{1}^{\perp}, j_{2}^{\perp}\right) \mapsto\left(j_{1}, j_{2}, j_{2}^{\perp}, j_{1}^{\perp}\right)$. It is plain that for no choice of $\varphi:\{1,2\} \rightarrow\{1,2\}$ and isomorphism $T_{k}$ can the map $T$ be approximated such that $T f(\varphi(k))-T_{k}(f(k))$ is small for all $k \in\{1,2\}$ and $f$ with $\|f\|=1$ (since $T f(\varphi(k))$ can be large with $f(k)$ small). Therefore $X$ (and more generally every $X$ with a nontrivial $M$-summand) fails to have the strong isomorphic Banach-Stone property.

6. Isomorphisms which are close to isometries. Let $X$ be a Banach space with $\alpha(X)=0$ and $K$ a compact space.

We choose an $\alpha>0$ and an admissible $\tau>0$ for this $\alpha$ (as in 2.3) and suppose that $T: C(K, X) \rightarrow C(K, X)$ is a $\tau$-isomorphism. We are going to investigate whether $T$ is close to an isometry. Let $\varphi$ be defined as in Theorem 2.4, and for simplicity we will assume for the moment that $\varphi=\operatorname{Id}_{K}$. Thus $T$ is a $\tau$-isomorphism with

$$
f(k)=0 \Rightarrow\|(T f)(k)\| \leq \alpha\|f\| .
$$

We consider maps $T_{k}: X \rightarrow X, x \mapsto(T \mathbf{x})(k)$, where $\mathbf{x}$ denotes the constant function $x$ (the $T_{k}$ are just the $S_{k}$ of $\S 2$, where $h=1$ ). Then, as it is shown in $\S 2$, the $T_{k}$ are isomorphisms on $X$ with

$$
\left\|T_{k}\right\| \leq 1+\tau, \quad\left\|T_{k}^{-1}\right\| \leq \frac{1+\tau}{1-2(1+\tau) \alpha} .
$$

Now consider the operator $\prod T_{k}$.

Since $k \mapsto T_{k}$ is continuous with respect to the strong operator topology (this can not be guaranteed in the general situation of $\$ 2$ ) this operator is well-defined on $C(K, X)$. 
Suppose it would be possible to find isometries $I_{k}$ on $X$ with $T_{k} \approx I_{k}$ and $k \mapsto I_{k}$ strongly continuous (so that $\prod I_{k}$ would be a well-defined isometry on $C(K, X))$. Then $T \approx \Pi T_{k}$ and $\Pi T_{k} \approx \Pi I_{k}$, so that $T$ would be close to an isometry.

Thus the main difficulty in this approximation problem is to approximate a continuous family of near-isometries continuously by isometries.

To treat this more precisely it is useful to introduce the following

6.1. Definition. Let $X$ be a Banach space and $\eta>0$. By $\operatorname{Iso}_{\eta}(X)$ we mean the collection of all isomorphisms $T: X \rightarrow X$ which are $\eta$-isomorphisms, i.e. $(1 /(1+\eta))\|x\| \leq\|T x\| \leq(1+\eta)\|x\|$.

We suppose that for every $\varepsilon>0$ there is an $\eta>0$ with

$$
\text { Iso }_{\eta}(X) \subset\left(\operatorname{IsO}_{0}(X)\right)_{\varepsilon},
$$

which is certainly true for finite-dimensional spaces (note that $\bigcap_{\eta>0} \operatorname{Iso}_{\eta}(X)=\operatorname{Iso}_{0}(X)$ and apply a compactness argument).

Then returning to the preceding discussion, we could choose $I_{k} \in$ Iso $_{0}(X)$ for every $k$ such that $\left\|T_{k}-I_{k}\right\| \leq \varepsilon$ (provided that $\tau$ was so small that $\left.T_{k} \in \mathrm{Iso}_{\eta}(X)\right)$. There seems, however, to be no general strategy to guarantee that the choice of these $I_{k}$ leads to a continuous family $k \mapsto I_{k}$.

We restrict ourselves by giving a sufficient condition:

6.2. Theorem. Let $X$ be a Banach space with $\alpha(X)=0$. Further suppose that for every $\tilde{\varepsilon}>0$ there is an $\eta>0$ such that

(i) $\operatorname{Iso}_{\eta}(X) \subset\left(\operatorname{Iso}_{0}(X)\right)_{\tilde{\varepsilon}}$,

(ii) there is a mapping $\mu: \operatorname{Iso}_{\eta}(X) \rightarrow \operatorname{Iso}_{0}(X)$ which is continuous with respect to the strong operator topology and for which $\|\mu(R)-R\| \leq$ $2 \tilde{\varepsilon}$ for every $R$.

Then $C(K, X)$ has the AI-property for every compact space $K$.

Proof. Let $\varepsilon>0$ be given. We choose $\mu>0$ and $\eta$ for $\tilde{\varepsilon}:=\varepsilon / 3$.

Now we select $\alpha>0$ with

$$
1+\alpha \leq 1+\eta, \quad \frac{1+\alpha}{1-2 \alpha(1+\alpha)} \geq \frac{1}{1+\eta}, \quad 2 \alpha \leq \frac{\varepsilon}{3}
$$

and finally $\tau>0$ with $\tau \leq \alpha$ for this $\alpha$ as in Definition 2.3. Let $T_{0}$ be a $\tau$-isomorphism on $C(K, X)$. We define $T:=T_{0} \circ I_{\varphi}$, where $\varphi$ is the homeomorphism defined by $T_{0}$ and $I_{\varphi}$ denotes the isometry $f \mapsto f \circ \varphi^{-1}$ on $C(K, X)$. Then $T$ is a $\tau$-isomorphism and we have $k \underset{T}{\stackrel{\alpha}{\longrightarrow}} k$ for every $k$. 
Consider the $T_{k}$ as above. Then $T_{k} \in \operatorname{Iso}_{\eta}(X)$ by construction, and $k \mapsto I_{k}:=\mu\left(T_{k}\right)$ is a continuous family (continuous w.r.t. the strong operator topology) of isometries such that $\left\|\Pi T_{k}-\Pi I_{k}\right\| \leq \frac{2}{3} \varepsilon$. But $\left\|T f(k)-T_{k}(f(k))\right\| \leq 2 \alpha\|f\|$ (since $\left.(f-\mathbf{f}(\mathbf{k}))(k)=0\right)$ so that $\| T-$ $\prod T_{k} \| \leq 2 \alpha \leq \varepsilon / 3$. This proves that $\left\|T-\prod I_{k}\right\|=\left\|T_{0}-\left(\prod I_{k}\right) I_{\varphi}^{-1}\right\| \leq$ $\varepsilon$, and $\left(\prod I_{k}\right)\left(I_{\varphi}\right)^{-1}$ is an isometry on $C(K, X)$.

Therefore $C(K, X)$ has the AI-property.

It seems to be difficult to find examples. We only mention the following

6.3. Theorem. Let $X$ be a finite-dimensional Banach space such that there are isometries $I_{1}, \ldots, I_{n}$ with $\operatorname{Iso}_{0}(X)=\cup_{i}\left\{\Theta I_{i}|| \Theta \mid=1\right\}$. (This is for example the case if the unit ball of $X$ has only finitely many extreme points.) Further assume that $\operatorname{Mult}(X)$ is trivial so that $\alpha(X)=0$.

Then $C(K, X)$ has the AI-property for every compact space $K$.

Proof. We first note: For every $I$ with $\|I\|=1$ and $\eta>0$ there is a continuous map

$$
\mu_{I}:(\{\Theta I|| \Theta \mid=1\})_{\eta} \rightarrow\{\Theta I|| \Theta \mid=1\}
$$

with $\left\|\mu_{I}(R)-R\right\| \leq 2 \eta$ for every $R$ (this is trivial if $\mathbf{K}=\mathbf{R}$ and easy if $\mathbf{K}=\mathbf{C}$ ).

It is now immediate how Theorem 6.2 can be applied: choose a minimal number of $I_{1}, \ldots, I_{n}$ and consider only such $\tilde{\varepsilon}$ where the $\left(\left\{\Theta I_{i}|| \Theta \mid=1\right\}\right)_{\tilde{\varepsilon}}$ are pairwise disjoint so that $\mu$ can be defined by gluing together the $\mu_{I_{i}}$.

As an important special case we mention the case $X=\mathbf{K}$. Here, however, it is easy to be more explicit, and one obtains

6.4. COROllary. For every $\tau$-isomorphism $T$ on a $C K$-space there is an isometry $I$ with $\|T-I\| \leq 3\left(\tau+\tau^{2}\right)$.

Finally it should be noted that there seems to be no possibility to treat similarly the case of $C_{0}(K, X)$ with locally compact $K$. The difficulties arise from the fact that in the definition of the $T_{k}$ we have to choose an element $h$ of $C_{0}(K)$ which is one at $k$ and this is not the same $h$ for all $k$ in the non-compact case. Of course something can be said if one has partitions of unity, but nothing up to now for 
arbitrary $K$. It is even not obvious how to treat the case $X=\mathbf{K}$ in general. If $\mathbf{K}=\mathbf{R}$ one has an analogue of Corollary 6.4 for arbitrary $C_{0} K$-spaces; the case $\mathbf{K}=\mathbf{C}$ however leads to the following problem: Let $K$ be locally compact and $T: C_{0} K \rightarrow C_{0} K$ a $\tau$-isomorphism. Suppose that for every compact $D \subset K$ there is a continuous function $h_{D}: D \rightarrow\{\Theta|| \Theta \mid=1\}$ with $\left\|\left.T f\right|_{D}-\left.h_{D} f\right|_{D}\right\| \leq \varepsilon\|f\|$ for every $f$. Is it possible to find a continuous $h: K \rightarrow\{\Theta|| \Theta \mid=1\}$ with $\|T f-h f\| \leq 2 \varepsilon\|f\|$ for all $f$ ?

Acknowledgments. I am grateful to the referee for drawing my attention to Jarosz' paper [17]. Globevnik's paper [14] was made available to me by D. Werner. The results proved there-together with our theorems-have the surprising consequence that all complex $L^{1}$-spaces have the strong isomorphic Banach-Stone property.

\section{REFERENCES}

[1] E. M. Alfsen and E. G. Effros, Structure in real Banach spaces I, II, Ann. of Math., 96 (1972), 98-173.

[2] D. Amir, On isomorphisms of continuous function spaces, Israel J. Math., 3 (1965), 205-210.

[3] E. Behrends, On the Banach-Stone theorem, Math. Annalen, 233 (1978), 261272.

[4] _ M-Structure and The Banach-Stone Theorem, Lecture Notes in Mathematics 736, Springer-Verlag 1979.

[5] How to obtain vector-valued Banach-Stone theorems by using $M$-structure methods, Math. Annalen, 261 (1982), 387-398.

[6] Multiplier representations and an application to the problem whether $A \otimes_{\varepsilon} X$ determines $A$ and/or $X$, Math. Scand., 52 (1983), 117-144.

[7] E. Behrends and M. Cambern, On isomorphic Banach-Stone theorems, (to appear: Studia Math.).

[8] Y. Benyamini, Small into-isomorphisms between spaces of continuous functions, Proc. Amer. Math. Soc., 83 (1981), 479-485.

[9] M. Cambern, On isomorphisms with small bound, Proc. Amer. Math. Soc., 18 (1967), 1062-1066.

[10] , Isomorphisms of $C_{0}(Y)$ onto $C(X)$, Pacific J. Math., 35 (1970), 307312.

[11] _ Isomorphisms of spaces of norm-continuous functions, Pacific J. Math., 116 (1985), 243-254.

[12] H. B. Cohen, $A$ bound-two isomorphism between $C(X)$ Banach spaces, Proc. Amer. Math. Soc., 50 (1975), 215-217.

[13] M. M. Day, Normed Linear Spaces, 3rd ed., Springer-Verlag, 1973.

[14] J. Globevnik, On complex strict and uniform convexity, Proc. Amer. Math. Soc., 47 (1975), 175-178. 
[15] K. Jarosz, A generalization of the Banach-Stone theorem, Studia Math., 73 (1982), 33-39.

[16] - Multipliers in complex Banach spaces and structure of the unit ball, preprint.

[17] - Perturbations of Banach Algebras, Lecture Notes in Mathematics 1120, Springer-Verlag 1985.

[18] J. Lindenstrauss and L. Tzafriri, Classical Banach Spaces I, Springer-Verlag, 1977.

[19] G. Wodinski, Multiplikatoren in komplexen Banachräumen, Dissertation, Freie Universität Berlin, 1986.

Received April 29, 1987 and in revised form August 10, 1987.

I. Mathematisches Institut

DER FREIEN UNIVERSITÄT BERLIN

ARNIMALLEE 3

D 1000 BERLIN 33

Federal Republic of Germany 


\title{
PACIFIC JOURNAL OF MATHEMATICS
}

\section{EDITORS}

\author{
V. S. VARADARAJAN \\ (Managing Editor) \\ University of California \\ Los Angeles, CA 90024 \\ HERBERT ClEMENS \\ University of Utah \\ Salt Lake City, UT 84112 \\ R. FINN \\ Stanford University \\ Stanford, CA 94305
}

\author{
HERMANN FLASCHKA \\ University of Arizona \\ Tucson, AZ 85721
}

RAMESh A. GANGOLLI University of Washington Seattle, WA 98195

VAUGHAN F. R. JONES University of California

Berkeley, CA 94720

\author{
ROBION KIRBY \\ University of California \\ Berkeley, CA 94720 \\ C. C. MOORE \\ University of California \\ Berkeley, CA 94720 \\ HAROLD STARK \\ University of California, San Diego \\ La Jolla, CA 92093
}

\section{ASSOCIATE EDITORS}

\author{
R. ARENS \\ E. F. BECKENBACH \\ B. H. NEUMANN \\ F. WOLF \\ K. YOSHIDA \\ (1906-1982)

\section{SUPPORTING INSTITUTIONS}

\begin{abstract}
UNIVERSITY OF ARIZONA
UNIVERSITY OF BRITISH COLUMBIA

UNIVERSITY OF CALIFORNIA

MONTANA STATE UNIVERSITY

UNIVERSITY OF NEVADA, RENO

NEW MEXICO STATE UNIVERSITY

OREGON STATE UNIVERSITY
\end{abstract} \\ CALIFORNIA INSTITUTE OF TECHNOLOGY \\ UNIVERSITY OF OREGON \\ UNIVERSITY OF SOUTHERN CALIFORNIA \\ STANFORD UNIVERSITY \\ UNIVERSITY OF HAWAII \\ UNIVERSITY OF TOKYO \\ UNIVERSITY OF UTAH \\ WASHINGTON STATE UNIVERSITY \\ UNIVERSITY OF WASHINGTON
}

The Supporting Institutions listed above contribute to the cost of publication of this Journal, but they are not owners or publishers and have no responsibility for its content or policies.

Mathematical papers intended for publication in the Pacific Journal of Mathematics should be in typed form or offset-reproduced (not dittoed), double spaced with large margins. Please do not use built up fractions in the text of the manuscript. However, you may use them in the displayed equations. Underline Greek letters in red, German in green, and script in blue. The first paragraph must be capable of being used separately as a synopsis of the entire paper. In particular it should contain no bibliographic references. Please propose a heading for the odd numbered pages of less than 35 characters. Manuscripts, in triplicate, may be sent to any one of the editors. Please classify according to the scheme of Math. Reviews, Index to Vol. 39. Supply name and address of author to whom proofs should be sent. All other communications should be addressed to the managing editor, or Elaine Barth, University of California, Los Angeles, California 90024.

There are page-charges associated with articles appearing in the Pacific Journal of Mathematics. These charges are expected to be paid by the author's University, Government Agency or Company. If the author or authors do not have access to such Institutional support these charges are waived. Single authors will receive 50 free reprints; joint authors will receive a total of 100 free reprints. Additional copies may be obtained at cost in multiples of 50 .

The Pacific Journal of Mathematics is issued monthly as of January 1966. Regular subscription rate: $\$ 190.00$ a year (5 Vols., 10 issues). Special rate: $\$ 95.00$ a year to individual members of supporting institutions.

Subscriptions, orders for numbers issued in the last three calendar years, and changes of address should be sent to Pacific Journal of Mathematics, P.O. Box 969, Carmel Valley, CA 93924, U.S.A. Old back numbers obtainable from Kraus Periodicals Co., Route 100, Millwood, NY 10546.

The Pacific Journal of Mathematics at P.O. Box 969, Carmel Valley, CA 93924 (ISSN 0030-8730) publishes 5 volumes per year. Application to mail at Second-class postage rates is pending at Carmel Valley, California, and additional mailing offices. Postmaster: send address changes to Pacific Journal of Mathematics, P.O. Box 969, Carmel Valley, CA 93924.

\section{PUBLISHED BY PACIFIC JOURNAL OF MATHEMATICS, A NON-PROFIT CORPORATION}




\section{Pacific Journal of Mathematics}

\section{Vol. 133, No. 2 \\ April, 1988}

William Charles Bauldry, Attila Mate and Paul Nevai, Asymptotics for solutions of systems of smooth recurrence equations . . . . . . . . . . 209

Ehrhard Behrends, Isomorphic Banach-Stone theorems and isomorphisms which are close to isometries ............................229

Fernanda Maria Botelho, Rotation sets of maps of the annulus .........251

Edward Graham Evans, Jr. and Phillip Alan Griffith, Binomial behavior

of Betti numbers for modules of finite length . ................. 267

Andrei Iordan, Pseudoconvex domains with peak functions at each point of

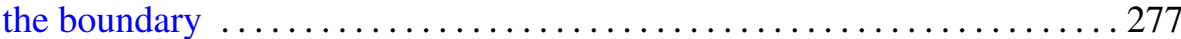

Zyun'iti Iwase, Dehn-surgery along a torus $T^{2}$-knot $\ldots \ldots \ldots \ldots \ldots \ldots . \ldots 289$

Marko Kranjc, Embedding 2-complexes in $\mathbf{R}^{4} \ldots \ldots \ldots \ldots \ldots \ldots \ldots \ldots \ldots$

Aloys Krieg, Eisenstein-series on real, complex, and quaternionic

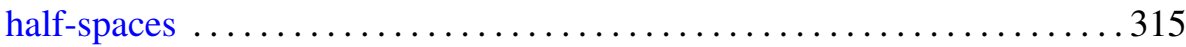

Masato Kuwata, Intersection homology of weighted projective spaces and

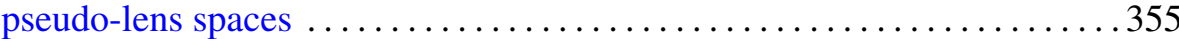

Carl Pomerance, András Sárközy and Cameron Leigh Stewart, On

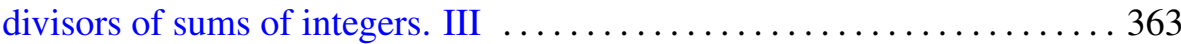

Martin Schechter, Potential estimates in Orlicz spaces ............... 381 